\title{
Functions on discrete sets holomorphic in the sense of Isaacs, or monodiffric functions of the first kind
}

\author{
Christer O. Kiselman
}

Department of Mathematics, Uppsala University, P. O. Box 480, SE-751 06 Uppsala, Sweden

(email: kiselman@math.uu.se)

Received December 10, 2004

\begin{abstract}
We study discrete analogues of holomorphic functions of one and two variables, especially those that were called monodiffric functions of the first kind by Rufus Isaacs. Discrete analogues of the Cauchy-Riemann operators, domains of holomorphy in one discrete variable, and the Hartogs phenomenon in two discrete variables are investigated.
\end{abstract}

Keywords: monodiffric functions of the first kind, discrete holomorphic functions, functions holomorphic in the sense of Isaacs, discrete Cauchy-Riemann operators, domains of holomorphy, Hartogs' phenomenon.

DOI: 10.1360/05za0006

\section{Introduction}

Discrete mathematics exhibits many phenomena analogous to those of classical analysis of real and complex variables. Topics like harmonic functions on $\mathbb{Z}^{n}$ received attention already eighty years ago. The study of holomorphic functions on $\mathbb{Z}^{2}$ has a history of more than sixty years.

The pioneer in the latter field is Rufus Isaacs, who introduced two difference equations, both of which are discrete counterparts of the Cauchy-Riemann equation in one complex variable. He thus defined two classes of holomorphic functions on the Gaussian integers $\mathbb{Z}[i]$, called monodiffric functions of the first and second kind, respectively ${ }^{[1]}$. In a later paper ${ }^{[2]}$ he pursued the study of the monodiffric functions of the first kind. More recent research on this class includes that of Nakamura \& Rosenfeld ${ }^{[3]}$.

Ferrand $^{[4]}$ investigated the monodiffric functions of the second kind, which she called préholomorphes (preholomorphic). Later studies on this class include those of Duffin ${ }^{[5]}$, Lovász $^{[6]}$, Kenyon ${ }^{[7]}$, and Benjamini \& Lovász ${ }^{[8]}$.

The purpose of this paper is to prove some results for functions defined on discrete sets which are analogous to those of holomorphic functions of one or several complex variables. Except for the definitions in Section 2 and brief remarks in Sections 3 and 4, we shall consider only monodiffric functions of the first kind. In particular we shall study 
the Cauchy-Riemann equation in one variable and the overdetermined system of CauchyRiemann equations in two variables (Section 4). The Hartogs phenomenon in two complex variables has a counterpart in the discrete setting (Section 6). Somewhat different is the situation concerning domains of holomorphy: only very special domains are domains of holomorphy in one discrete variable (Section 5).

\section{Functions defined on polygons}

Let $\Gamma$ be a closed polygon in the complex plane $\mathbb{C}$ consisting of $m$ edges $\left[a_{0}, a_{1}\right]$, $\left[a_{1}, a_{2}\right], \ldots,\left[a_{m-1}, a_{0}\right]$, where $a_{0}, \ldots, a_{m-1}$ are given points in $\mathbb{C}$. The polygon is thus determined by $\left(a_{0}, \ldots, a_{m-1}\right) \in \mathbb{C}^{m}$. We shall say that a complex-valued function $f$ defined on $\Gamma$ is piecewise affine if $f$ is affine on each segment $\left[a_{j}, a_{j+1}\right]$ with the possible exception of the points that belong to two or more segments. This means that, if $a_{j+1} \neq$ $a_{j}$

$f(z)=\frac{a_{j+1}-z}{a_{j+1}-a_{j}} f\left(a_{j}\right)+\frac{z-a_{j}}{a_{j+1}-a_{j}} f\left(a_{j+1}\right), \quad z \in\left[a_{j}, a_{j+1}\right], \quad j=0, \ldots, m-1$,

except for the finitely many points belonging to some other segment $\left[a_{k}, a_{k+1}\right]$. Here and in the sequel we count indices modulo $m$. If $a_{j+1}=a_{j}$ the formula reduces to $f(z)=f\left(a_{j}\right)=f\left(a_{j+1}\right)$.

The integral of a piecewise affine function is easy to calculate.

Proposition 2.1. Let $f$ be piecewise affine on a closed polygon $\Gamma$ determined by $\left(a_{0}, \ldots, a_{m-1}\right)$. Then

$$
\int_{\Gamma} f(z) d z=\frac{1}{2} \sum_{0}^{m-1} f\left(a_{j}\right)\left(a_{j+1}-a_{j-1}\right)=\frac{1}{2} \sum_{0}^{m-1}\left(f\left(a_{j-1}\right)-f\left(a_{j+1}\right)\right) a_{j} .
$$

Proof. If $f$ is affine on a segment $\left[a_{j}, a_{j+1}\right]$ we can replace it by its average over the segment without changing the value of the integral. The average is $\frac{1}{2} f\left(a_{j}\right)+\frac{1}{2} f\left(a_{j+1}\right)$. This implies that

$$
\begin{aligned}
\int_{a_{j}}^{a_{j+1}} f(z) d z & =\int_{a_{j}}^{a_{j+1}}\left(\frac{1}{2} f\left(a_{j}\right)+\frac{1}{2} f\left(a_{j+1}\right)\right) d z \\
& =\left(\frac{1}{2} f\left(a_{j}\right)+\frac{1}{2} f\left(a_{j+1}\right)\right)\left(a_{j+1}-a_{j}\right) .
\end{aligned}
$$

To finish we just need to sum over $j$ and change the indices.

Definition 2.2. Given a closed polygon $\Gamma$ in $\mathbb{C}$ we shall say that a function $f$ defined on its vertices is holomorphic on $\Gamma$ if $\int_{\Gamma} f_{\text {aff }}(z) d z=0$, where $f_{\text {aff }}$ is the unique piecewise affine function on $\Gamma$ which takes the same values as $f$ on the vertices.

Proposition 2.1 now yields the following result.

Corollary 2.3. Let $a_{0}, \ldots, a_{m-1}$ be $m$ points in the complex plane. A function defined on the vertices of the closed polygon $\Gamma$ defined by $\left(a_{0}, \ldots, a_{m-1}\right)$ is holomorphic 
on $\Gamma$ if and only if

$$
\sum_{0}^{m-1} f\left(a_{j}\right)\left(a_{j+1}-a_{j-1}\right)=0
$$

When $m=2$, every function is holomorphic.

When $m=3$ and $\left(a_{0}, a_{1}, a_{2}\right)=(a, b, c)$, the condition becomes

$$
f(a)(b-c)+f(b)(c-a)+f(c)(a-b)=0,
$$

which can be written as

$$
\frac{f(b)-f(a)}{b-a}=\frac{f(c)-f(a)}{c-a} .
$$

This means that the difference quotient is the same in the direction from $a$ to $b$ as in the direction from $a$ to $c$. In particular, if $b=a+1$ and $c=a+i$, we get

$$
\frac{f(a+1)-f(a)}{1}=\frac{f(a+i)-f(a)}{i} .
$$

Definition 2.4. A complex-valued function $f$ defined on a subset $A$ of $\mathbb{Z}[i]$ shall be said to be holomorphic in the sense of Isaacs or monodiffric of the first kind if (2.4) holds for all $a \in A$ such that also $a+1$ and $a+i$ belong to $A$.

When $m=4$ and $\left(a_{0}, a_{1}, a_{2}, a_{3}\right)=(a, b, c, d)$, condition (2.2) becomes

$$
f(a)(b-d)+f(b)(c-a)+f(c)(d-b)+f(d)(a-c)=0,
$$

which may be written

$$
\frac{f(c)-f(a)}{c-a}=\frac{f(d)-f(b)}{d-b},
$$

meaning that the difference quotient in the direction from $a$ to $c$ is equal to that in the direction from $b$ to $d$. This is the definition studied by Ferrand ${ }^{[4]}$. In particular, if we let $b=a+1, c=a+1+i$, and $d=a+i$, we get

$$
\frac{f(a+1+i)-f(a)}{1+i}=\frac{f(a+i)-f(a+1)}{i-1} .
$$

Definition 2.5. A function $f$ defined on a subset $A$ of $\mathbb{Z}^{2}=\mathbb{Z}[i]$ shall be said to be holomorphic in the sense of Ferrand or monodiffric of the second kind if (2.6) holds for all $a \in A$ such that also $a+1, a+i$, and $a+1+i$ all belong to $A$.

In this paper we shall study functions which are holomorphic in the sense of Isaacs. We shall compare them with the classical theory of holomorphic functions of one or several complex variables. Indeed we may identify the grid $\mathbb{Z}^{2}$ with the Gaussian integers $\mathbb{Z}[i]=\mathbb{Z}+i \mathbb{Z}$ and regard it as a subset of the complex plane $\mathbb{C}$.

Concerning the history of these concepts and our choice of terms, let us note that Rufus Isaacs ${ }^{[1]}$ introduced functions defined on the grid $\mathbb{Z}^{2}$ which satisfy (2.4); he called them monodiffric of the first kind. In a later paper ${ }^{[2]}$ he called them just monodiffric. He called the functions defined on $\mathbb{Z}^{2}$ which satisfy (2.6) monodiffric of the second kind ${ }^{[1]}$. That class was further studied by Jacqueline Ferrand ${ }^{[4]}$. Isaacs, however, expressed the opinion 
that they "seemed less promising than the present course" (i.e., the study of monodiffric functions of the first kind) ${ }^{[2, \mathrm{p} .258]}$. I therefore think it is justifiable to associate the monodiffric functions of the first kind with the name of Isaacs, and those of the second kind with the name of Ferrand.

\section{Cauchy-Riemann operators}

The Cauchy-Riemann operator that corresponds to the first definition of Isaacs is

$$
(\operatorname{cr} f)(z)=f(z+1)+i f(z+i)-(1+i) f(z), \quad z \in \mathbb{Z}[i] .
$$

A function $f: A \rightarrow \mathbb{C}$, where $A$ is a subset of $\mathbb{Z}[i]$, is holomorphic in $A$ in the sense of Isaacs (Definition 2.4) if and only if $\operatorname{cr}(f)(z)=0$ at all points $z$ such that $z, z+1, z+i \in$ $A$. This means that $f$ solves a convolution equation $\mu * f=0$ in $A \cap(A-1) \cap(A-i)$, where $\mu=\delta_{-1}+i \delta_{-i}-(1+i) \delta_{0}$. We shall write $f \in \mathcal{O}(A)$.

This definition of a holomorphic function is not invariant under rotation by $90^{\circ}$ or $180^{\circ}$. However, it behaves well under the reflection $z \mapsto i \bar{z}$ in $\mathbb{Z}[i]$, which just interchanges the real and imaginary parts: $(x, y) \mapsto(y, x)$ in $\mathbb{Z}^{2}$. Let us write $z^{\text {refl }}=i \bar{z}$ and $A^{\text {refl }}$ for the set of all $z^{\text {refl }}$ with $z \in A$. The following result is easy to prove.

Lemma 3.1. $\quad$ Let $f$ be defined on a subset $A$ of $\mathbb{Z}[i]$ and define $g(z)=\overline{f(i \bar{z})}$ for $z$ in the reflected domain $A^{\text {refl }}$. Then $(\operatorname{crg} g)(z)=i \overline{(\operatorname{cr} f)(i \bar{z})}$. Hence $f$ is holomorphic if and only if $g$ is holomorphic in the reflected domain.

Example 3.2. An exponential function

$$
E_{a, b}(z)=a^{x} b^{y}=e^{\alpha x+\beta y}, \quad x+i y \in \mathbb{Z}[i],
$$

is holomorphic if and only if

$$
\operatorname{cr}\left(E_{a, b}\right)(x+i y)=a^{x} b^{y}(a+i b-1-i)=0,
$$

i.e., precisely when $b=1-i+i a$. We thus have a one-parameter family of holomorphic exponential functions $h_{c}(z)=(1+c)^{x}(1+i c)^{y}, z \in \mathbb{Z}[i], c \in \mathbb{C}$. Of all functions with $|a|=1$, thus bounded on the real axis, the one with fastest growth as $y \rightarrow+\infty$ is obtained when $a=(-1-i) / \sqrt{2}$ and $b=(\sqrt{2}+1)(1-i) / \sqrt{2}$,

$$
f(x+i y)=\left(\frac{-1-i}{\sqrt{2}}\right)^{x}\left((\sqrt{2}+1) \frac{1-i}{\sqrt{2}}\right)^{y},
$$

and the one with fastest decay as $y \rightarrow+\infty$ is obtained when $a=(1+i) / \sqrt{2}$ and $b=(\sqrt{2}-1)(1-i) / \sqrt{2}$,

$$
f(x+i y)=\left(\frac{1+i}{\sqrt{2}}\right)^{x}\left((\sqrt{2}-1) \frac{1-i}{\sqrt{2}}\right)^{y} .
$$

In the first case we have $|f(x+i y)|=(\sqrt{2}+1)^{y}$; in the second $|f(x+i y)|=(\sqrt{2}-1)^{y}$, $y \in \mathbb{Z}$.

Another Cauchy-Riemann operator, which is more symmetric, is

$$
\mathrm{CR}(f)(z)=f(z+1)-f(z-1)+i f(z-1)-i f(z-i) .
$$


Also this operator was introduced by Isaacs ${ }^{[1, \text { p.179] }}$ Ferrand $^{[4]}$ studied it in other coordinates: instead of the points $1, i,-1,-i$ she used $1+i,-1+i,-1-i, 1-i$. The two operators are essentially the same; it suffices to rotate the plane by $45^{\circ}$ and make a change of scale.

The companion operator is

$$
\left(\mathrm{CR}^{*} f\right)(z)=f(z+1)-f(z-1)-i f(z-1)+i f(z-i) .
$$

The two operators map functions defined on the pure points (the points whose real and imaginary parts have the same parity) to functions defined on the mixed points (the points whose real and imaginary parts are of different parity) - we denote them by $\mathrm{CR}_{p}, \mathrm{CR}_{p}^{*}$. Conversely $\mathrm{CR}_{m}$ and $\mathrm{CR}_{m}^{*}$ map functions on mixed points to functions on pure points. We note that $\mathrm{CR}_{m} \circ \mathrm{CR}_{p}^{*}=\Delta_{p}$, the Laplacian defined on the pure points,

$$
\left(\Delta_{p} f\right)(z)=f(z+2)+f(z-2)+f(z+2 i)+f(z-2 i)-4 f(z) .
$$

We refer to ref. [9] and the references mentioned there for more on the discrete Laplacian.

\section{The Cauchy-Riemann equations}

Theorem 4.1. There exist three fundamental solutions $E_{j}, j=1,2,3$, to cr with support respectively in

$$
\begin{gathered}
A_{1}=\{z \in \mathbb{Z}[i] ; 1-\operatorname{Re} z \leqslant \operatorname{Im} z \leqslant 0\}, \\
A_{2}=A_{1}^{\text {refl }}=\{z \in \mathbb{Z}[i] ; 1-\operatorname{Im} z \leqslant \operatorname{Re} z \leqslant 0\},
\end{gathered}
$$

and

$$
A_{3}=\{z \in \mathbb{Z}[i] ; \operatorname{Re} z \leqslant 0, \operatorname{Im} z \leqslant 0\} .
$$

Proof. Define $E_{1}(z)=0$ when $\operatorname{Re} z \leqslant 0 ; E_{1}(1)=1$ (so that $\left(\operatorname{cr} E_{1}\right)(0)=1$ ), and $E_{1}(1+i y)=0$ for $y \neq 0$. Then define recursively $E_{1}(z)$ when $\operatorname{Re} z=p+1, p \geqslant 1$, so that the Cauchy-Riemann equation with zero right-hand side is satisfied for $\operatorname{Re} z=p$. This is easy: the equation $\operatorname{cr}\left(E_{1}\right)(p+i y)=0$ says that

$$
E_{1}(p+1+i y)+i E_{1}(p+i(y+1))-(1+i) E_{1}(p+i y)=0,
$$

so we just solve for $E_{1}(p+1+i y)$. It is then easy to see that $E_{1}(p+1+i y)$ vanishes when $y \geqslant 1$ or $y \leqslant-p-1$. We have $\operatorname{cr} E_{1}=\delta_{0}$. The proofs for $E_{2}$ and $E_{3}$ are similar.

Isaacs $^{[1, \text { p.197] }}$ constructed these functions.

Theorem 4.2 $2^{[1]}$. Given any function $f$ on $\mathbb{Z}[i]$, the equation $\operatorname{cr} u=f$ can be solved.

Proof. Let $E_{j}$ be the fundamental solution with support in $A_{j}, j=1,2$, constructed in Theorem 4.1. We denote by $\chi$ the characteristic function of the half plane $\{z ; \operatorname{Re} z \geqslant \operatorname{Im} z\}$. Then we can form the convolution products in the formula

$$
u=E_{1} *(\chi f)+E_{2} *(1-\chi) f
$$

and apply the Cauchy-Riemann operator and then use the associative law as follows:

$$
\begin{aligned}
\operatorname{cr} u & =\mu * u=\mu *\left(E_{1} *(\chi f)\right)+\mu *\left(E_{2} *(1-\chi) f\right) \\
& =\left(\mu * E_{1}\right) *(\chi f)+\left(\mu * E_{2}\right) *((1-\chi) f)=\chi f+(1-\chi) f=f .
\end{aligned}
$$


We remark that convolution, defined by

$$
(f * g)(z)=\sum_{w \in \mathbb{Z}[i]} f(w) g(z-w), \quad z \in \mathbb{Z}[i],
$$

is in general not an associative binary operation. A simple example can be found even in $\mathbb{Z}$ : let $h=\chi_{\mathbb{N}}$ be the characteristic function of $\mathbb{N}$, let $g=\delta_{0}-\delta_{1}$, and let $f=a h+b$ for some constants $a, b$. Then $(f * g) * h=a \delta_{0} * h=a h$, while $f *(g * h)=f * \delta_{0}=a h+b$. Associativity holds only if $b=0$. Here $f * g$ and $g * h$ are well defined, but $f * h$ is not, except when $b=0$. However, in (4.1) the support of the fundamental solutions is contained in proper cones, and not only $E_{1} *(\chi f)$ and $\mu * E_{1}$ but also $\mu *(\chi f)$ are well defined: the sums defining each convolution are finite, and the associative law holds.

Duffin $^{[5, \text { p.348] }}$ solved the equation CR $u=f($ cf. (3.2)) in $\mathbb{Z}[i]$ using Fourier methods, but only if $f$ is in $l^{1}(\mathbb{Z}[i])$, i.e., if $\sum_{z \in \mathbb{Z}[i]}|f(z)|$ is finite.

Theorem 4.3. A system of equations $\mathrm{cr}_{1} u=f_{1}, \mathrm{cr}_{2} u=f_{2}$, where the $f_{j}$ are given in $\mathbb{Z}[i]^{2}$, can be solved if and only if $\mathrm{cr}_{2} f_{1}=\mathrm{cr}_{1} f_{2}$.

Proposition 4.4. An equation $\mathrm{cr}_{2} v=g$, where $g$ is given in $\mathbb{Z}[i]^{2}$, can be solved with $v$ holomorphic in $z_{1}$ if and only if $g$ is holomorphic in $z_{1}$.

Proof. We construct for each fixed $z_{1}$ the solution in the variable $z_{2}$ as in (4.1) and observe that it is holomorphic in $z_{1}$ if $g$ is. Indeed, using (4.1),

$$
\mathrm{cr}_{1} u=\mu_{1} * u=E_{1} *\left(\chi\left(\mu_{1} * g\right)\right)+E_{2} *\left((1-\chi)\left(\mu_{1} * g\right)\right)=0 .
$$

The calculation is justified by the fact that $\mu_{1}$ has its support in the plane $z_{2}=0$ and $\chi$ is a function of $z_{2}$, so that $\mu_{1} *(\chi g)=\chi\left(\mu_{1} * g\right)$.

Proof of Theorem 4.3. We first solve $\mathrm{cr}_{1} w=f_{1}$ for each $z_{2}$. Now a new unknown function $v=u-w$ satisfies $\mathrm{cr}_{1} v=0$ if and only if $\mathrm{cr}_{1} u=f_{1}$. And $\mathrm{cr}_{2} v=\mathrm{cr}_{2} u-$ $\mathrm{cr}_{2} w=f_{2}-\mathrm{cr}_{2} w$ if and only if $\mathrm{cr}_{2} u=f_{2}$. So we need to solve $\mathrm{cr}_{1} v=0$ and $\mathrm{cr}_{2} v=$ $f_{2}-\mathrm{cr}_{2} w=g$. This we can do using the proposition, for $g$ is holomorphic in $z_{1}: \mathrm{cr}_{1} g=$ $\mathrm{cr}_{1} f_{2}-\mathrm{cr}_{1} \mathrm{cr}_{2} w=\mathrm{cr}_{2}\left(f_{1}-\mathrm{cr}_{1} w\right)=0$ in view of the condition $\mathrm{cr}_{1} f_{2}=\mathrm{cr}_{2} f_{1}$ and the choice of $w$.

\section{Domains of holomorphy}

Given two subsets $A$ and $B$ of $\mathbb{Z}[i]$ with $A \subset B$ we have a restriction operator $R_{A}^{B}: \mathcal{O}(B) \rightarrow \mathcal{O}(A)$. It may well be that $R_{A}^{B}$ is injective but not surjective, or surjective but not injective.

Example 5.1. Let $A=\{0,1\}, B=A \cup\{i\}$. Then $R$ is bijective.

Example 5.2. Let $A=\{0, i, 1+i, 2+i, 2\}, B=A \cup\{1\}$. Then $R$ is easily seen to be injective, but it is not surjective, for a holomorphic function on $B$ must satisfy

$$
(1+i) f(0)-i f(i)=f(1)=\frac{1}{1+i}(f(2)+i f(1+i)) .
$$


Its restriction to $A$ must then satisfy

$$
(1+i) f(0)-i f(i)=\frac{1}{1+i}(f(2)+i f(1+i)) .
$$

But any function on $A$ is holomorphic.

Example 5.3. Let $A=\{0,1, i\}$ and $B=A \cup\{2\}$. Then the restriction mapping is surjective but not injective.

In view of the properties of the restriction operator $R_{A}^{B}$ it seems reasonable to propose the following definition.

Definition 5.4. A domain of holomorphy in $\mathbb{Z}[i]$ is a set $A$ such that if $B \supset A$ and $R_{A}^{B}$ is bijective, then $B=A$.

However, in analogy with the situation in $\mathbb{C}^{n}, n \geqslant 2$, it may be of interest to admit also Riemann domains over $\mathbb{Z}[i]$ and $\mathbb{Z}[i]^{n}$. Blanc ${ }^{[10]}$ introduced such non-schlicht domains, although he did not define domains of holomorphy. Let us only mention here that questions on domains of holomorphy receive different answers in the two settings. The set

$$
\{z ; \operatorname{Re} z \geqslant 0, \operatorname{Im} z \geqslant 0, \operatorname{Re} z+\operatorname{Im} z \leqslant 3\} \backslash\{(1,1)\}
$$

is a domain of holomorphy in the sense of Definition 5.4, but not if we allow non-schlicht domains. A theory of holomorphic functions on the vertices and their dual vertices on a Riemann surface has been developed by Mercat ${ }^{[11,12]}$.

Let us call a set $A$ 8-connected if any two points in $A$ can be joined by a path consisting of vertical, horizontal and diagonal segments, all with endpoints in $A$. We call $A$ 4-connected if only vertical and horizontal segments are allowed. Between these two connectivity properties we have $6 /$-connectedness and $6 \backslash$-connectedness. In the first, vertical and horizontal segments and northeast-southwest diagonals are allowed; in the second, vertical and horizontal segments and northwest-southeast diagonals are allowed. (We should perhaps call them north-south, east-west, northeast-southwest, and northwestsoutheast segments in order not to mix the two ways of naming directions.)

To any set $A$ in $\mathbb{Z}[i]$ we associate the smallest triangle $T(A)$ of the form

$$
\{z ; \operatorname{Re} z \geqslant a, \operatorname{Im} z \geqslant b, \operatorname{Re} z+\operatorname{Im} z \leqslant c\}
$$

which contains it. (We allow $-a,-b, c \in \mathbb{Z} \cup\{+\infty\}$.)

Example 5.5. The set $A=\{0,1+i, 2+i, 1+2 i\}$ is $6 /$-connected but not $6 \backslash$-connected. The kernel of the restriction operator $R_{A}^{T(A)}$ has dimension 1; the cardinality of $T(A) \backslash A$ is 6 .

Example 5.6. The set $A=\{i, 2 i, 1+i, 2\}$ is $6 \backslash$-connected but not $6 /$-connected. The restriction operator $R_{A}^{T(A)}$ is a bijection.

As is clear from these examples, one should preferably represent points $(x, y) \in \mathbb{Z}^{2}$ not by $x+i y$ but by $x+\omega y \in \mathbb{C}$, where $\omega=\frac{1}{2}+\frac{1}{2} \sqrt{3} i$. Then $6 \backslash$-connectedness becomes the natural connectedness, while 6/-connectedness looks strange. The supports 
of the three fundamental solutions also look much better in this representation: each is a sector of opening $60^{\circ}$.

Theorem 5.7. Let $A \subset \mathbb{Z}[i]$ be $6 \backslash$-connected and such that $A \cap L$ is an interval whenever $L$ is a line $\operatorname{Re} z=$ const., $\operatorname{Im} z=$ const., or $\operatorname{Re} z+\operatorname{Im} z=$ const. Then every $f \in \mathcal{O}(A)$ can be uniquely extended to the smallest triangle of the form (5.1) which contains $A$.

This result is not optimal, for there are sets which do not satisfy the hypotheses and still admit a unique continuation to the triangle.

Proof. The smallest triangle

$$
T(A)=\left\{z ; \operatorname{Re} z \geqslant a_{0}, \operatorname{Im} z \geqslant b_{0}, \operatorname{Re} z+\operatorname{Im} z \leqslant c_{0}\right\}
$$

which contains an 8 -connected set $A$ is the union of three sets,

$$
\begin{gathered}
{[(A+i \mathbb{N}) \cap(A+(-1+i) \mathbb{N})] \cup[(A+\mathbb{N}) \cap(A+(1-i) \mathbb{N})]} \\
\cup[(A-\mathbb{N}) \cap(A-i \mathbb{N})] .
\end{gathered}
$$

We shall prove that any holomorphic function on $A$ can be uniquely extended to $(A+i \mathbb{N}) \cap(A+(-1+i) \mathbb{N})$ by successively extending the function to larger and larger sets. The key step is the following lemma.

Lemma 5.8. Given a bounded set $A$ as in Theorem 5.7 and any $6 \backslash$-connected proper subset $B$ of $(A+i \mathbb{N}) \cap(A+(-1+i) \mathbb{N})$ containing $A$ and with the properties that $B \cap L$ is an interval for any horizontal or vertical line as well as a line with equation $\operatorname{Re} z+\operatorname{Im} z=$ const. and to which any holomorphic function on $A$ can be uniquely continued, we can find a strictly larger set $B^{\prime}$ with the same properties.

Proof. Let $\{b, b+1, \ldots, b+m-1\}$ be the points in $B$ with maximal imaginary part. Here $m$, the number of points with largest imaginary part, is at least 1 . In the construction of $B^{\prime}$ we shall distinguish between the following cases.

Case 1: $m \geqslant 2$. We take $B^{\prime}=B \cup\{b+i, b+1+i, \ldots, b+m-2+i\}$.

Case 2: $m=1, \operatorname{Re} b>a_{0}$. There exists a point $c \in B$ with $\operatorname{Re} c=\operatorname{Re} b-1$. Take $c$ with maximal imaginary part, so that $c+i \notin B$. Define $B^{\prime}=B \cup\{c+i\}$. Note that $c+1$ must belong to $B$; this follows from the $6 \backslash$-connectedness. The fact that both $c$ and $c+1$ belong to $B$ guarantees the uniqueness of the extension to $c+i$. (It is not enough that $c+1+i$ belongs to $B$. Note that $\{c, c+1+i\}$ is $6 /$-connected but not $6 \backslash$-connected. The proof would break down at this point if we had used 6/-connectedness; cf. Example 5.5.)

Case 3: $m=1, \operatorname{Re} b+\operatorname{Im} b<c_{0}$. There exists a point $c \in B$ with $\operatorname{Re} c+\operatorname{Im} c=$ $\operatorname{Re} b+\operatorname{Im} b+1$. Take $c$ with maximal imaginary part, so that $c-1+i \notin B$. Define $B^{\prime}=B \cup\{c-1+i\}$. The point $c-1$ must belong to $B$, which guarantees the uniqueness of the continuation to $c-1+i$. (Here 8 -connectedness suffices.)

In these three cases it is easy to check that the new set $B^{\prime}$ has all properties needed. 
Case 4: $m=1, \operatorname{Re} b=a_{0}$ and $\operatorname{Re} b+\operatorname{Im} b=c_{0}$. This means that $b$ is the top point of $T(A)$ and it follows that $B=(A+i \mathbb{N}) \cap(A+(-1+i) \mathbb{N})$.

Proof of Theorem 5.7 (continued). We construct $A_{1}=A^{\prime}, A_{j+1}=A_{j}^{\prime}, j \in \mathbb{N}^{*}$. After a while we arrive at case 4 in the proof of Lemma 5.8, which implies that $A_{j}=$ $(A+i \mathbb{N}) \cap(A+(-1+i) \mathbb{N})$.

The result for $(A+\mathbb{N}) \cap(A+(1-i) \mathbb{N})$ follows by reflection, and the proof for $(A-\mathbb{N}) \cap(A-i \mathbb{N})$ is similar. This concludes the proof for bounded $A$.

For an unbounded set $A$ we define

$$
A^{k}=\{z \in A ; \operatorname{Re} z \geqslant-k, \operatorname{Im} z \geqslant-k, \operatorname{Re} z+\operatorname{Im} z \leqslant k\} .
$$

Then $A^{k}$ is bounded and satisfies all hypotheses of the theorem, so any holomorphic function on $A^{k}$ can be uniquely continued to the triangle $T\left(A^{k}\right)$. Hence any holomorphic function on $\bigcup A^{k}=A$ can be uniquely continued to $\bigcup T\left(A^{k}\right)=T(A)$.

Corollary 5.9. A $6 \backslash$-connected subset of $\mathbb{Z}[i]$ such that the intersection $A \cap L$ is an interval whenever $L$ is a line $\operatorname{Re} z=$ const., $\operatorname{Im} z=$ const., or $\operatorname{Re} z+\operatorname{Im} z=$ const. is a domain of holomorphy if and only if $A=T(A)$.

Remark 5.10. Any set $A$ is a disjoint union of maximal $6 \backslash$-connected sets $A_{j}$. A function $f \in \mathcal{O}\left(A_{j}\right)$ can be extended to $T\left(A_{j}\right)$, but it may of course happen that $T\left(A_{j}\right)$ intersects some other $6 \backslash$-connectivity component $A_{k}, k \neq j$.

If $\Omega$ is a rectangle in $\mathbb{Z}[i]$, say defined by $a_{0} \leqslant \operatorname{Re} z \leqslant a_{1}$ and $b_{0} \leqslant \operatorname{Im} z \leqslant b_{1}$, we define

$$
\begin{aligned}
b^{\prime} \Omega & =\left\{z \in \Omega ; \operatorname{Re} z=a_{1} \text { or } \operatorname{Im} z=b_{0}\right\}, \\
b^{\prime \prime} \Omega & =\left\{z \in \Omega ; \operatorname{Re} z=a_{0} \text { or } \operatorname{Im} z=b_{1}\right\}, \\
b \Omega & =b^{\prime} \Omega \cup b^{\prime \prime} \Omega .
\end{aligned}
$$

The set $b \Omega$ will serve as a kind of boundary of $\Omega$ in the following.

A holomorphic function defined on a triangle

$$
\{z \in \mathbb{Z}[i] ; \operatorname{Re} z \geqslant a, \operatorname{Im} z \geqslant b, \operatorname{Re} z+\operatorname{Im} z \leqslant c\}
$$

for some $a, b, c \in \mathbb{Z}$ is determined by its restriction to any of the three sides of the triangle. Also any function defined on a side of the triangle can be extended uniquely to a holomorphic function on the whole triangle. (This follows from Theorem 5.7, but is easy to prove directly.) Using this result, we see that a holomorphic function on a rectangle $\Omega$ is determined by its restriction to $b^{\prime} \Omega$; likewise by its restriction to $b^{\prime \prime} \Omega$. Indeed there are formulas

$$
h(z)=\sum_{s \in b^{\prime} \Omega} C_{\Omega}(z, s) h(s), \quad z \in \Omega, \quad h \in \mathcal{O}(\Omega)
$$

and

$$
h(z)=\sum_{s \in b^{\prime \prime} \Omega} C_{\Omega}(z, s) h(s), \quad z \in \Omega, \quad h \in \mathcal{O}(\Omega)
$$


where $C_{\Omega}(\cdot, s) \in \mathcal{O}(\Omega)$ for every $s \in b \Omega$.

Conversely, if $f$ is any function defined on $b^{\prime} \Omega$, then the formula

$$
h(z)=\sum_{s \in b^{\prime} \Omega} C_{\Omega}(z, s) f(s), \quad z \in \Omega,
$$

defines a holomorphic function $h$ on $\Omega$. Formula (5.2) gives rise to an estimate,

$$
|h(z)| \leqslant \sup _{s \in b^{\prime} \Omega}|h(s)| \sum_{s \in b^{\prime} \Omega}\left|C_{\Omega}(z, s)\right|, \quad z \in \Omega, \quad h \in \mathcal{O}(\Omega),
$$

which, however, is very bad. Nevertheless, $h \in \mathcal{O}(\Omega)$ is determined by $\left.h\right|_{b^{\prime} \Omega}$.

Let us take a look at another extension problem: take $A=\mathbb{Z}$ and $B=\mathbb{Z}[i]$. Then it is easy to see that any function $f$ on $A$ can be extended uniquely as a holomorphic function $F$ in the upper half plane $\mathbb{Z}+i \mathbb{N}$. The extension satisfies an estimate

$$
|F(x+i y)| \leqslant(\sqrt{2}+1)^{y} \sup _{0 \leqslant x^{\prime} \leqslant x}\left|f\left(x^{\prime}\right)\right|, \quad(x, y) \in \mathbb{Z}+i \mathbb{N} .
$$

In particular, if $f$ is bounded, then $F$ is of exponential type.

On the other hand, there are infinitely many ways to extend $f$ to the lower half plane, and there is no estimate on the growth. In fact, given arbitrary functions $g:-\mathbb{N}^{*} \rightarrow \mathbb{C}$ and $\varphi:-\mathbb{N}^{*} \rightarrow \mathbb{Z}$, there is a unique holomorphic extension $G$ to the lower half plane $\mathbb{Z}-i \mathbb{N}$ satisfying $G(\varphi(y)+i y)=g(y)$ for all $y \in-\mathbb{N}^{*}$. (We write $\mathbb{N}^{*}$ for $\mathbb{N} \backslash\{0\}$.) Here $g$ may grow as fast as we like. Thus holomorphic extension from the real axis $\mathbb{Z}$ to the whole Gaussian plane $\mathbb{Z}[i]$ is always possible, but questions on uniqueness and bounds have highly asymmetric answers.

\section{The Hartogs phenomenon}

In several complex variables it is known that a holomorphic function cannot have its singularities contained in a compact set. More precisely, the following theorem describes how a holomorphic function can be extended over a hole.

Theorem 6.1. Let $\Omega$ be an open set in $\mathbb{C}^{n}, n \geqslant 2$, and $K$ a compact subset of $\Omega$. If $h \in \mathcal{O}(\Omega \backslash K)$, then there exists a function $H \in \mathcal{O}(\Omega)$ such that $H=h$ in $\Omega \backslash L$ for some compact $L \subset \Omega$.

There is a similar phenomenon in $\mathbb{Z}[i]^{2}$. We formulate it in the simplest case only.

Theorem 6.2. Let $\Omega=\Omega_{1} \times \Omega_{2}$, where $\Omega_{j}$ are two rectangles in $\mathbb{Z}[i]$, and let $K \subset \Omega$ be a subset which does not meet $\left(b \Omega_{1} \times \Omega_{2}\right) \cup\left(\Omega_{1} \times b \Omega_{2}\right)$. If $h \in \mathcal{O}(\Omega \backslash K)$, then there exists a function $H \in \mathcal{O}(\Omega)$ such that $H=h$ in $\Omega \backslash L$ for some set $L$ not intersecting $\left(b \Omega_{1} \times \Omega_{2}\right) \cup\left(\Omega_{1} \times b \Omega_{2}\right)$.

Proof. Construct a function $H$ by the formula

$$
H\left(z_{1}, z_{2}\right)=\sum_{s_{1} \in b^{\prime} \Omega_{1}} C_{\Omega}\left(z_{1}, s_{1}\right) h\left(s_{1}, z_{2}\right), \quad z=\left(z_{1}, z_{2}\right) \in \Omega
$$

cf. (5.4). Clearly $H$ is holomorphic in $z_{1}$ (since $C_{\Omega}$ is), and holomorphic in $z_{2}$ (since $h$ is).

Copyright by Science in China Press 2005 
First fix $z_{2} \in \Omega_{2} \backslash \pi_{2}(K)$, where $\pi_{2}$ is the projection $\left(z_{1}, z_{2}\right) \mapsto z_{2}$. Then $H\left(z_{1}, z_{2}\right)$ $=h\left(z_{1}, z_{2}\right)$ for all $z_{1} \in \Omega_{1}$; cf. (5.2).

Next fix $z_{1} \in \Omega_{1} \backslash \pi_{1}(K)$. We know that $H\left(z_{1}, z_{2}\right)=h\left(z_{1}, z_{2}\right)$ when $z_{2} \in \Omega_{2} \backslash$ $\pi_{2}(K)$, in particular when $z_{2} \in b^{\prime} \Omega_{2}$. In view of the uniqueness of holomorphic continuation, we must have $H\left(z_{1}, z_{2}\right)=h\left(z_{1}, z_{2}\right)$ for all $z_{2} \in \Omega_{2}$.

Summing up, we have proved that $H$ is holomorphic in $\Omega$ and that $H=h$ in $\Omega_{1} \times$ $\left(\Omega_{2} \backslash \pi_{2}(K)\right)$ as well as in $\left(\Omega_{1} \backslash \pi_{1}(K)\right) \times \Omega_{2}$. We may thus take $L$ as $\pi_{1}(K) \times \pi_{2}(K)$, a set which does not meet $\left(b \Omega_{1} \times \Omega_{2}\right) \cup\left(\Omega_{1} \times b \Omega_{2}\right)$.

Is it possible to take a smaller $L$ ? In other words, how far can the equality $H=h$ be extended? This can be described using the unique extension to triangles discussed in Section 5.

\section{References}

1. Isaacs, R. P., A finite difference function theory, Univ. Nac. Tucumán. Revista A, 1941, 2: 177—201.

2. Isaacs, R. P., Monodiffric functions, in: Construction and Applications of Conformal Maps, Proceedings of a Symposium, National Bureau of Standards Appl. Math. Series., No. 18, Washington, D. C.: U. S. Government Printing Office, 1952, 257-266.

3. Nakamura, A., Rosenfeld, A., Digital calculus, Inform. Sci., 1997, 98: 93-98.

4. Ferrand, J., Fonctions préharmoniques et fonctions préholomorphes, Bull. Sci. Math., 1944, 68: 152-180.

5. Duffin, R. J., Basic properties of discrete analytic functions, Duke Math. J., 1956, 23: 335-363.

6. Lovász, L., Discrete Analytic Functions: an Exposition, Manuscript, 2000, 1-46, available at http://research. microsoft.com/users/lovasz/analytic.pdf

7. Kenyon, R., Conformal invariance of domino tiling, Ann. Probab., 2000, 28: 759-795.

8. Benjamini, I., Lovász, L., Harmonic and analytic functions on graphs, J. Geom., 2002, 76: 2-15.

9. Kiselman, C. O., Subharmonic functions on discrete structures, in: Harmonic Analysis, Signal Processing, and Complexity, Festschrift in Honor of the 60th Birthday of Carlos A. Berenstein, Progress in Mathematics, Boston: Birkhäuser, to appear.

10. Blanc, C., Les réseaux Riemanniens, Comment. Math. Helv., 1940-41, 13: 54-67.

11. Mercat, C., Holomorphie et modèle d'Ising, Thèse, Université Louis Pasteur, 1998, 1-144.

12. Mercat, C., Discrete Riemann surfaces and the Ising model, Comm. Math. Phys., 2001, 218(1): 177—216. 